\title{
Elevated sphingosine-1-phosphate promotes sickling and sickle cell disease progression
}

\author{
Yujin Zhang, ${ }^{1}$ Vladimir Berka, ${ }^{2}$ Anren Song, ${ }^{1}$ Kaiqi Sun, ${ }^{1,3}$ Wei Wang, ${ }^{1,4}$ Weiru Zhang, ${ }^{1,4}$ Chen Ning, ${ }^{1,5}$ \\ Chonghua Li, ${ }^{1}$ Qibo Zhang, ${ }^{6}$ Mikhail Bogdanov, ${ }^{1}$ Danny C. Alexander, ${ }^{6}$ Michael V. Milburn, ${ }^{6}$ \\ Mostafa H. Ahmed, 7 Han Lin, ${ }^{8}$ Modupe Idowu, ${ }^{2}$ Jun Zhang, ${ }^{9}$ Gregory J. Kato, ${ }^{10}$ \\ Osheiza Y. Abdulmalik, ${ }^{11}$ Wenzheng Zhang, ${ }^{2}$ William Dowhan, ${ }^{1}$ Rodney E. Kellems, ${ }^{1,3}$ \\ Pumin Zhang, ${ }^{8}$ Jianping Jin,, ${ }^{1,3}$ Martin Safo, ${ }^{7}$ Ah-Lim Tsai, ${ }^{2}$ Harinder S. Juneja, ${ }^{2}$ and Yang Xia ${ }^{1,3,4}$
}

${ }^{1}$ Department of Biochemistry and Molecular Biology, ${ }^{2}$ Department of Internal Medicine, and ${ }^{3}$ Graduate School of Biomedical Science, University of Texas Health Science Center at Houston, Houston, Texas, USA. ${ }^{4}$ Department of Nephrology and ${ }^{5}$ Department of Urology, The First Xiangya Hospital, Central South University, Changsha, Hunan, People's Republic of China. 6 Metabolon Inc., Durham, North Carolina, USA. 7Department of Medicinal Chemistry, School of Pharmacy, and Institute for Structural Biology and Drug Discovery, Virginia Commonwealth University, Richmond, Virginia, USA. ${ }^{8}$ Department of Physiology and ${ }^{9}$ Department of Hematology/Oncology, Baylor College of Medicine, Houston, Texas, USA. ${ }^{10}$ Sickle Cell Disease Division, National Heart, Lung and Blood Institute, NIH, Bethesda, Maryland, USA. ${ }^{11}$ Children's Hospital of Philadelphia, Philadelphia, Pennsylvania, USA.

\begin{abstract}
Sphingosine-1-phosphate (S1P) is a bioactive lipid that regulates multicellular functions through interactions with its receptors on cell surfaces. S1P is enriched and stored in erythrocytes; however, it is not clear whether alterations in $\mathrm{S} 1 \mathrm{P}$ are involved in the prevalent and debilitating hemolytic disorder sickle cell disease (SCD). Here, using metabolomic screening, we found that S1P is highly elevated in the blood of mice and humans with SCD. In murine models of SCD, we demonstrated that elevated erythrocyte sphingosine kinase 1 (SPHK1) underlies sickling and disease progression by increasing S1P levels in the blood. Additionally, we observed elevated SPHK1 activity in erythrocytes and increased S1P in blood collected from patients with SCD and demonstrated a direct impact of elevated SPHK1-mediated production of S1P on sickling that was independent of $\mathrm{S} 1 \mathrm{P}$ receptor activation in isolated erythrocytes. Together, our findings provide insights into erythrocyte pathophysiology, revealing that a SPHK1-mediated elevation of $\mathrm{S} 1 \mathrm{P}$ contributes to sickling and promotes disease progression, and highlight potential therapeutic opportunities for SCD.
\end{abstract}

\section{Introduction}

Sickle cell disease (SCD) is a devastating and costly genetic disorder associated with high morbidity and mortality. It is the most prevalent autosomal recessive disorder, affecting millions worldwide, with approximately 300,000 infants born each year with SCD $(1,2)$. Although the cellular and molecular bases of SCD have long been known (3-5), it is disappointing that we currently only have one FDA-approved drug, i.e., hydroxyurea, to treat SCD. This unfortunate situation results from a poor understanding of the molecular mechanisms underlying the initiation of sickling, the fundamental process of disease pathogenesis. Thus, identifying specific factors and signaling pathways involved in the initiation and propagation of sickling is essential to advance our understanding of this pathogenic process and develop novel strategies for the treatment of SCD.

Metabolite profiles accurately measure functional phenotypes that are the net result of genomic, transcriptomic, and proteomic changes (6). Therefore, metabolomic profiling provides one of the most integrated functional profiles of biologic status and is a robust analytical tool to discern mechanisms of action (7). Metabolomic profiling is especially appropriate for erythrocytes, in which gene expression profiling is not an option due to lack of a nucleus. Thus, comparison of metabolic profiles between erythrocytes of normal individuals and those with SCD is likely to identify functionally important differences that may provide improved understanding

Conflict of interest: The authors have declared that no conflict of interest exists. Citation for this article: J Clin Invest. 2014;124(6):2750-2761. doi:10.1172/JCI74604. of the sickling process. In this way, global metabolomic alterations in SCD are likely to reveal unrecognized biomarkers, deregulated pathways, and novel therapeutic possibilities. Using nonbiased high-throughput metabolomic profiling, here we report that sphingosine-1-phosphate (S1P), a bioactive lipid enriched in erythrocytes $(8,9)$, is significantly elevated in erythrocytes and plasma of mice and humans with SCD. S1P is an important signaling molecule, which regulates diverse biological processes, including inflammation (10), angiogenesis $(11,12)$, endothelial injury $(13)$, and thrombosis (14, 15) via activation of cell surface S1P receptors and/or by interaction with key regulatory proteins within cells (16). S1P is generated intracellularly by 2 enzymes: sphingosine kinase 1 and 2 (SPHK1 and SPHK2). Intracellular S1P levels are generally determined by a balance in the activities of S1P-synthesizing and -degrading enzymes. In most cells, the activities of the degrading enzymes are higher, keeping intracellular S1P levels low (10). Erythrocytes are unique among cells because they lack S1P-degrading enzymes. As a result, erythrocytes have high levels of S1P and are considered to be the major cell type for storing and supplying plasma S1P $(8,9)$. In addition, because human erythrocytes lack SPHK2, which localizes predominantly to the nucleus (17), only SPHK1 is used to generate S1P in human erythrocytes $(18,19)$. Although S1P is abundant in erythrocytes, the physiological role of S1P in erythrocytes remains unknown.

Here, using both pharmacologic and genetic approaches, we demonstrate that increased erythrocyte SPHK1 underlies elevated $\mathrm{S} 1 \mathrm{P}$ within erythrocytes and plasma in SCD mice and that elevated $\mathrm{S} 1 \mathrm{P}$ contributes to sickling and disease progression. Next, we validated our mouse studies using primary cultures of erythrocytes 
A All significant lipids: sorted by average $Z$

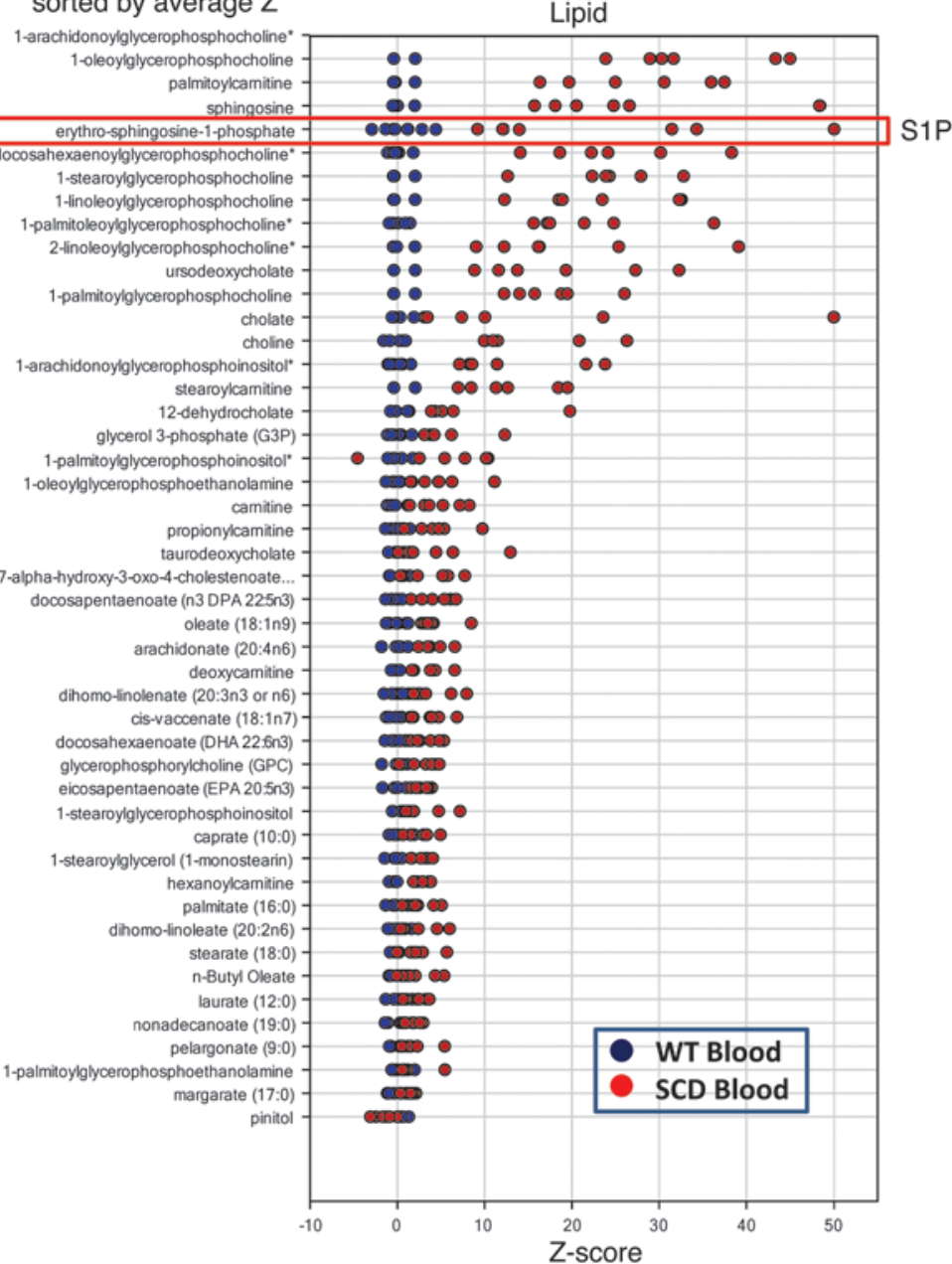

B

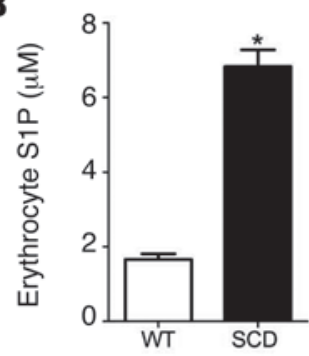

D

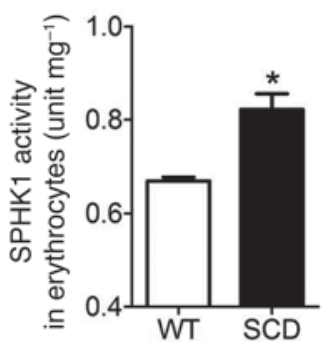

C

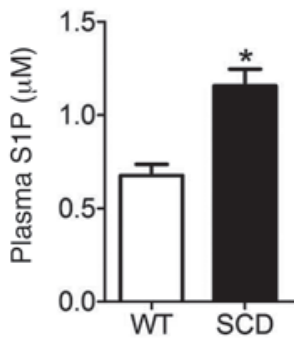

$\mathbf{E}$

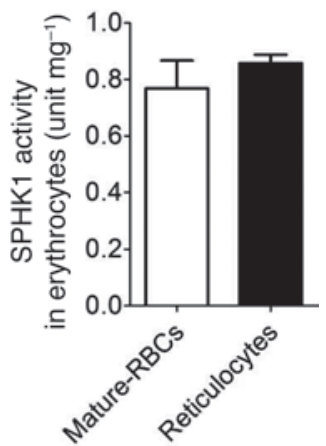

Figure 1

Metabolomic screening reveals that blood S1P levels and erythrocyte SPHK1 activity are elevated in SCD Tg mice. (A) Z-score quantification of lipids detected in the blood of both WT and SCD Tg mice. Among all lipids detected, S1P was one of the most substantially elevated in the blood of SCD Tg mice compared with that in WT mice $(n=6)$. (B and C) LC/MS POS platform measurement indicates that S1P levels in the (B) erythrocytes and (C) plasma of SCD mice were significantly elevated compared with those in WT mice $(n=6-8)$. (D) Total erythrocyte SPHK1 activity was significantly elevated in SCD Tg mice compared with that in WT mice $(n=8)$. (E) SPHK1 activities in purified reticulocytes and mature erythrocytes of SCD Tg mice. Values shown represent the mean $\pm \operatorname{SEM}(n=6-8)$. ${ }^{*} P<0.05$ versus WT.

from humans with SCD and showed a direct impact of elevated SPHK1-mediated induction of S1P on hypoxia-induced sickling independent of S1P receptor activation. Taken together, our studies identify a previously unrecognized role of S1P in sickling and innovative therapies for the disease.

\section{Results}

Metabolomic screening and biochemical analysis reveal that erythrocyte S1P and SPHK1 activity are elevated in SCD mice. To identify metabolic alterations associated with SCD, we conducted nonbiased metabolomic screening by using liquid and gas chromatography coupled with mass spectral analysis (LC/GC-MS) to measure and compare metabolite profiles in the whole blood of controls and Berkeley SCD Tg mice, a well-accepted animal model of SCD (20, 21). Of 7,000 metabolites screened, 251 metabolites were detected in the circulation of both WT and SCD Tg mice. Among these metabolites, we chose to focus on erythrocyte-specific metabolites. Initial screening revealed that $\mathrm{S} 1 \mathrm{P}$, a bioactive lipid enriched and stored in erythrocytes $(8,9)$, was one of the most elevated metabolites in the whole blood of SCD Tg mice compared with that in WT mice (Figure 1A). Subsequently, the results of the metabolic screen were confirmed using a sensitive mass spectral analysis and also revealed that $\mathrm{S} 1 \mathrm{P}$ levels were significantly elevated in both plasma and erythrocytes of SCD Tg mice $(P<0.003, n=6)$ (Figure $1, \mathrm{~B}$ and $\mathrm{C})$. Although S1P, a lipid signaling molecule, is enriched in erythrocytes $(8,9)$ and involved in multiple pathophysiological processes $(13,16,22)$, nothing is known about the role of S1P in erythrocyte physiology or its pathological role in SCD.

Because erythrocytes have no S1P-degrading enzymes $(8,9)$ and lack SPHK2 (17), only SPHK1 is used to generate S1P in human erythrocytes $(18,19)$. Thus, we hypothesized that SPHK1 is a key enzyme responsible for increased S1P generation seen in SCD mice. 


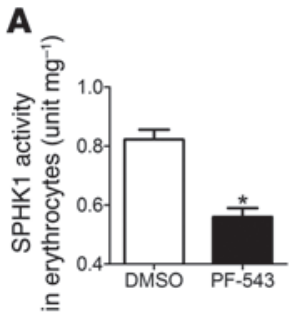

E

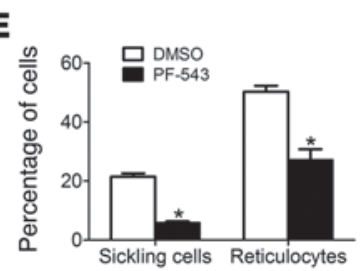

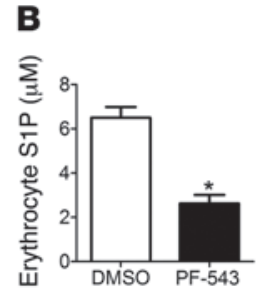
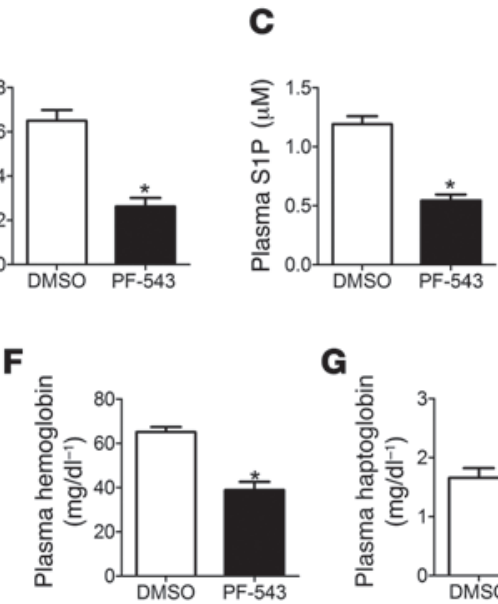

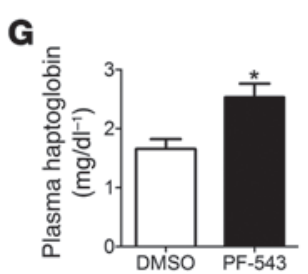

D
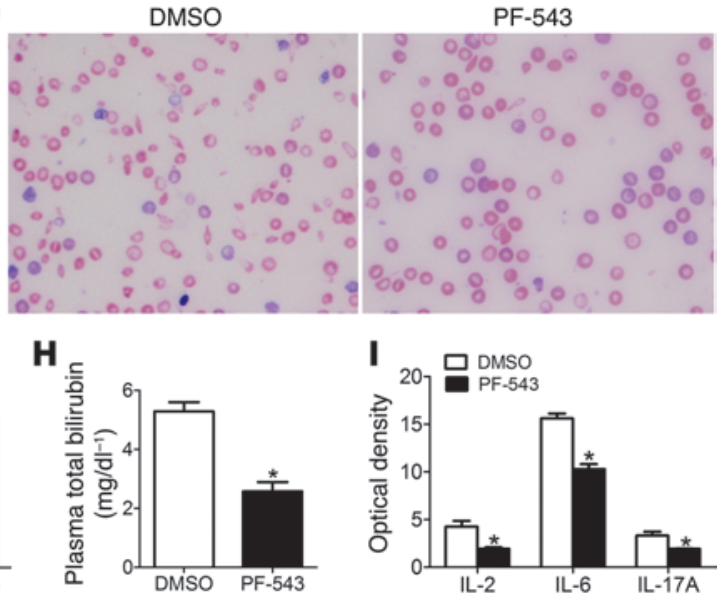

Figure 2

PF-543, a potent, specific SPHK1 inhibitor, reduces sickling, hemolysis, and inflammation in SCD Tg mice by reducing erythrocyte SPHK1 activity and S1P levels. (A-C) PF-543 treatment significantly reduced (A) erythrocyte SPHK1 activity, (B) erythrocyte levels, and (C) plasma levels of S1P in SCD Tg mice. (D) Representative blood smears of SCD Tg mice, as a function PF-543 treatment (original magnification, $\times 100$ ). (E) Percentages of sickle cells and reticulocytes were significantly reduced by PF-543 treatment in SCD Tg mice. (F-I) Effects of PF-543 treatment on (F) plasma $\mathrm{Hb},(\mathbf{G})$ plasma haptoglobin, (H) plasma total bilirubin, and (I) circulating cytokines in SCD Tg mice. Values shown represent the mean \pm SEM $(n=6-11) .{ }^{*} P<0.05$ versus SCD Tg mice treated with DMSO.

To address these possibilities, we first measured SPHK1 activity in the erythrocytes of normal and SCD mice. As expected, we found that SPHK1 activity was significantly elevated in the total erythrocytes isolated from SCD Tg mice compared with that in the erythrocytes from WT mice (Figure 1D). Realizing that SCD Tg mice contain a large population of young reticulocytes, it is possible that the elevated SPHK1 activity seen in SCD mouse erythrocytes may be due to increased reticulocytes in these mice. To examine this possibility, we compared SPHK1 activity in mature erythrocytes and young reticulocytes purified from SCD mice. We found that the SPHK1 activity in these two cell types was similar (Figure 1E). Thus, this finding demonstrates that SPHK1 activity is elevated in both mature and young erythrocytes in SCD mice.

Elevated erythrocyte SPHK1 activity underlies increased blood S1P levels and contributes to sickling in SCD mice. Next, to determine whether elevated SPHK1 activity is responsible for the increased S1P levels in SCD Tg mice, we initially took a pharmacologic approach. First, we compared the inhibitory effects on SPHK1 activity in cultured erythrocytes from SCD mice of two structurally different SPHK1 inhibitors, SK1-I (23) and PF-543 (24), a newly developed potent SPHK1 inhibitor. For this purpose, we cultured erythrocytes isolated from SCD mice under hypoxic conditions in the presence or absence of different concentrations of these 2 inhibitors. Unexpectedly, we found that SK1-I induced sickle cell hemolysis at a concentration of $5 \mu \mathrm{M}$ (Supplemental Figure 1A; supplemental material available online with this article; doi:10.1172/JCI74604DS1). In contrast, we did not observe hemolysis in PF-543-treated sickle cells at concentrations of up to $50 \mu \mathrm{M}$ (Supplemental Figure 1A). Furthermore, we found that both SK-1 $(<5 \mu \mathrm{M})$ and PF-543 inhibited SPHK1 activity and reduced S1P levels in cultured sickle cells in a dosage-dependent manner under hypoxic conditions (Supplemental Figure 1, B and C), while PF-543 showed much more potent inhibitory effects on SPHK1 activity compared with SK1-I (Supplemental Figure 1, B-E). Additionally, we found that treatment with both SK1-I $(<5 \mu \mathrm{M})$ and PF-543 decreased hypoxia-induced sickling in a dosage-dependent manner (Supplemental Figure 1, F and G). Of note, PF-543 showed much more potent antisickling effects compared with SK1-I (Supplemental Figure 1, F and G). Thus, these studies indicate that elevated SPHK1 activity underlies increased S1P and that elevated S1P is responsible for hypoxia-induced sickling in cultured SCD mouse erythrocytes.

Because PF-543 showed more potent in vitro inhibitory effects on SPHK1 activity and sickling and did not have the side effect of hemolysis, as did SK1-I, we chose to treat SCD Tg mice with PF-543 to determine the pathological significance of elevated SPHK1-mediated S1P production increases in vivo. Prior to treating SCD Tg mice with PF-543, we chose to treat WT mice with DMSO or PF-543 to assess toxicity. We did not see any obvious side effects on hematological parameters or tissue damage. Following our toxicity testing in WT mice, we proceeded to treat SCD mice with DMSO and PF-543 for 4 weeks to compare the efficacy. We found that PF-543 treatment significantly decreased erythrocyte SPHK1 activity of SCD Tg mice (Figure 2A). Additionally, we found that PF-543 treatment resulted in the reduction of erythrocyte and plasma S1P levels in SCD Tg mice (Figure 2 , B and C). Moreover, analysis of blood smears revealed that the shape of erythrocytes was much more uniform and organized in PF-543-treated SCD Tg mice (Figure 2D). Supporting this observation, we found that chronic treatment with PF-543 significantly reduced the percentages of sickled cells and reticulocytes in SCD Tg mice (Figure 2E). Thus, these findings provide pharmacologic evidence that increased SPHK1 activity is a key causative factor underlying increased S1P in erythrocytes and plasma of SCD Tg mice and contributes to sickling.

PF-543 treatment decreases intravascular hemolysis and reduces inflammation in SCD Tg mice. Because PF-543 treatment reduced sickling in SCD Tg mice, it is possible that chronic treatment with PF-543 also has a beneficial effect on intravascular hemolysis, a major complication associated with SCD (25). To test this hypothesis, we treated SCD Tg mice with PF-543 for 4 weeks to lower S1P levels 

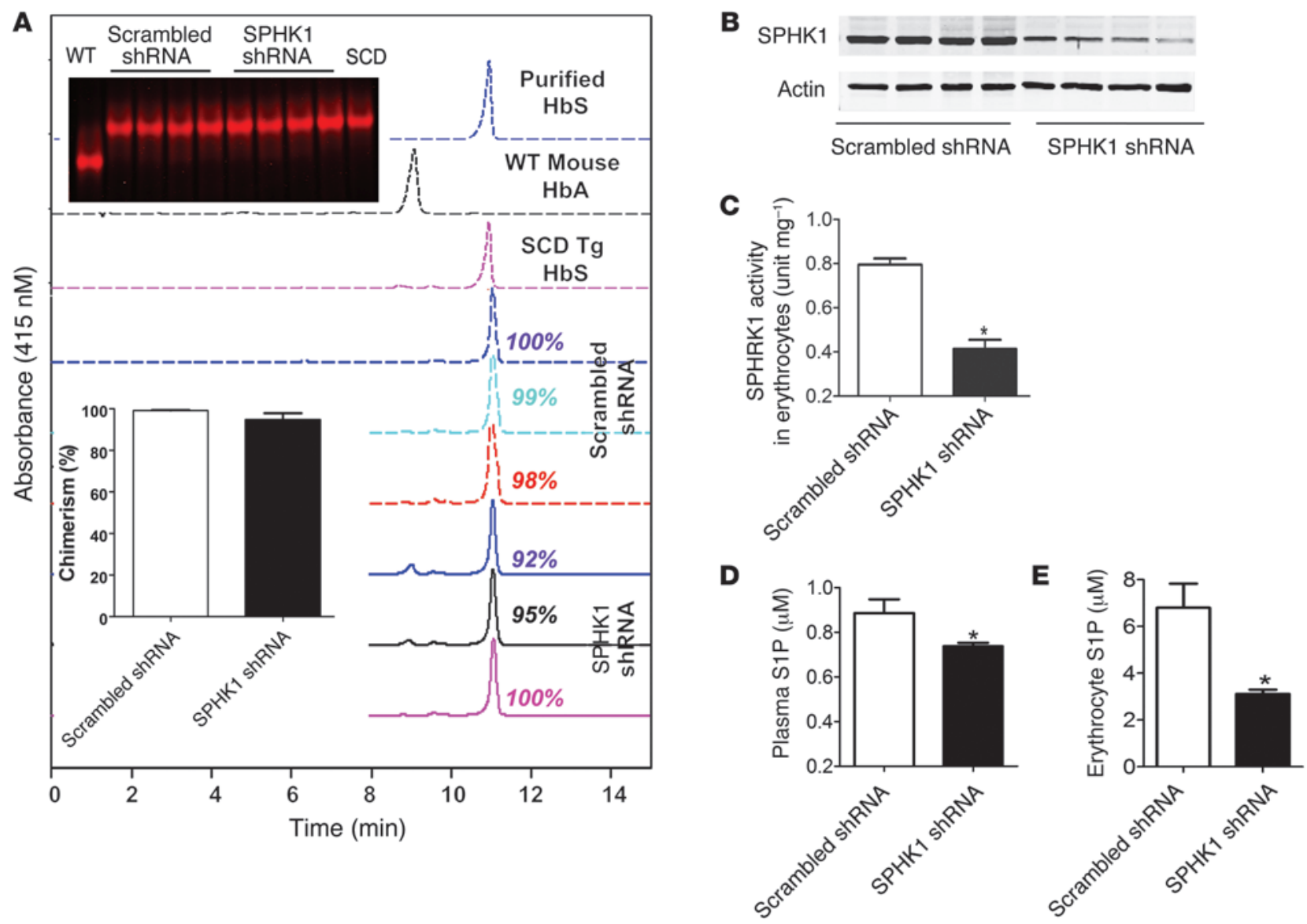

Figure 3

Specific knockdown of SPHK1 in HSCs in BMT SCD chimeras reduced erythrocyte SPHK1 protein levels and erythrocyte and plasma S1P levels. (A) HPLC and electrophoresis analysis of $\mathrm{HbS}$ and mouse normal $\mathrm{Hb}(\mathrm{HbA})$ in BMT SCD chimeras to assess the percentage of chimerism. Representative electrophoresis analysis and the average of percentage of chimerism with $\mathrm{HbS}$ in SCD chimeras were shown as insets. Data shown represent the mean \pm SEM $(n=6-9)$. (B) Erythrocyte SPHK1 levels, (C) activity, (D) plasma S1P levels, and (E) erythrocyte S1P levels were significantly reduced in the SCD chimeras with specific SPHK1 knockdown compared with those in mice with BMCs infected with a scrambled shRNA. ${ }^{\star} P<0.05$ versus SCD chimeras with BMCs infected with recombinant lentivirus encoding scrambled shrna.

and reduce erythrocyte sickling, as described above. Intravascular hemolysis in SCD Tg mice was significantly reduced by PF-543 treatment, as demonstrated by decreased plasma hemoglobin ( $\mathrm{Hb})$, increased plasma haptoglobin, and decreased total bilirubin (Figure $2, \mathrm{~F}-\mathrm{H})$. Because of the antisickling and antihemolytic effects of PF-543 treatment, complete blood count (CBC) analysis showed that PF-543 treatment significantly increased the total number of erythrocytes, $\mathrm{Hb}$ concentration, and hematocrit (Supplemental Table 1). Additionally, following treatment, the erythrocyte distribution width was significantly reduced, reflecting more uniformly shaped cells, as seen in blood smear (Figure 2D). These findings suggest that PF-543 treatment leads to decreased percentages of sickled cells and reticulocytes and thereby results in less hemolysis in SCD mice.

$\mathrm{S} 1 \mathrm{P}$ is a potent inflammatory stimulator $(10,13,16,26)$, and an increased inflammatory response is another major complication associated with sickling and hemolysis. Thus, it is possible that lowering S1P in SCD Tg mice may lead to a decreased inflammatory response. To test this possibility, we treated SCD Tg mice with PF-543 for 4 weeks, as described above. CBC analysis revealed that
PF-543 treatment reduced the total number of white blood cells to the normal range (Supplemental Table 1). Of note, both circulating lymphocytes and neutrophils were significantly reduced in PF-543-treated SCD mice (Supplemental Table 1). Moreover, we found that circulating inflammatory cytokines, including IL-2, IL-6, and IL-17A, were significantly reduced by PF-543 treatment in SCD mice (Figure 2I). Altogether, we provide in vivo evidence that PF-543 is an effective treatment to decrease sickling, reticulocyte count, hemolysis, and inflammation by reducing elevated SPHK1-mediated increased S1P levels.

PF-543 treatment attenuates tissue injury at multiple sites and splenomegaly and increases survival rates of SCD mice. Without intervention, chronic sickling, hemolysis, and inflammation will eventually lead to splenomegaly (an early feature associated with SCD to clear increased sickled cells), tissue injury at multiple sites, and early death. Histological studies revealed that chronic PF-543 treatment markedly reduced splenic damage, congestion, and necrosis (Supplemental Figure 2A). As such, we found that the splenomegaly was significantly reduced in PF-543-treated mice compared with DMSO-treated SCD mice (Supplemental Fig- 
A

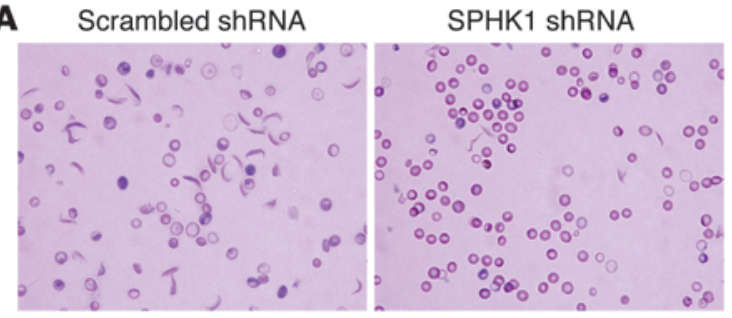

B
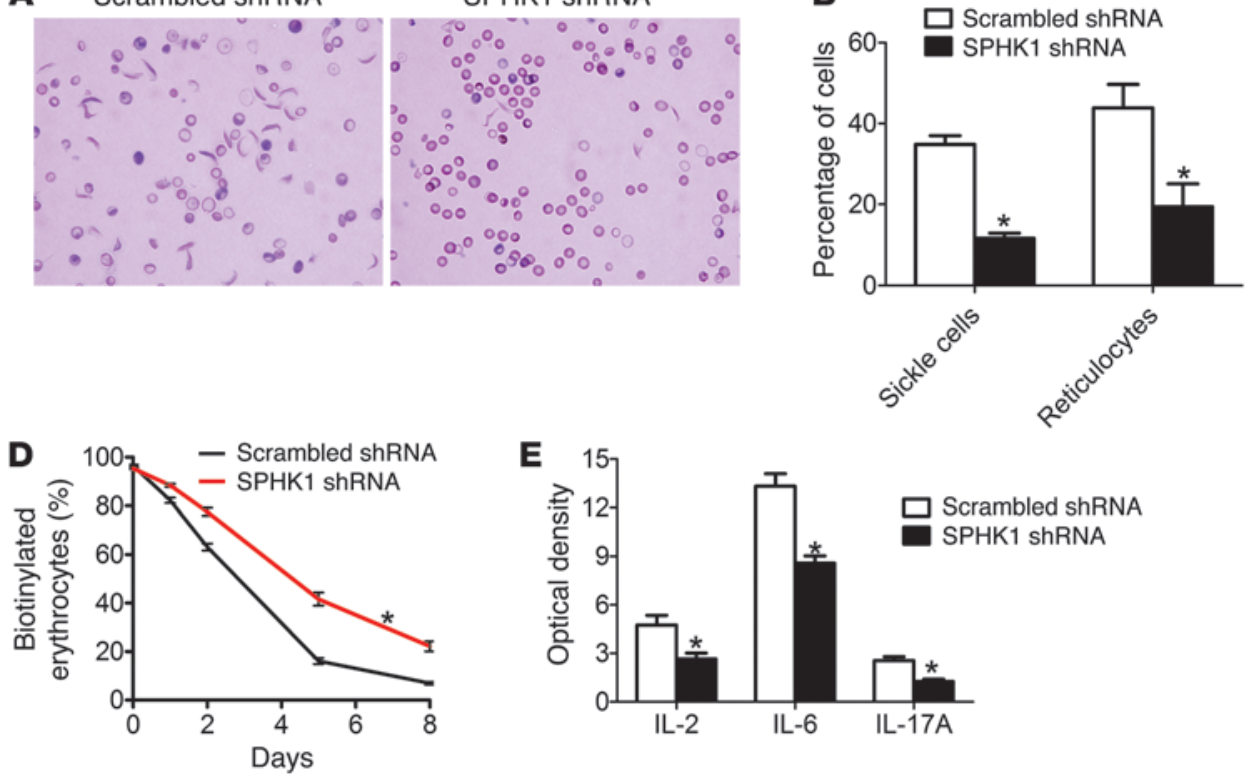

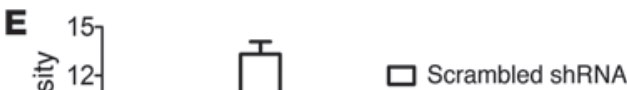
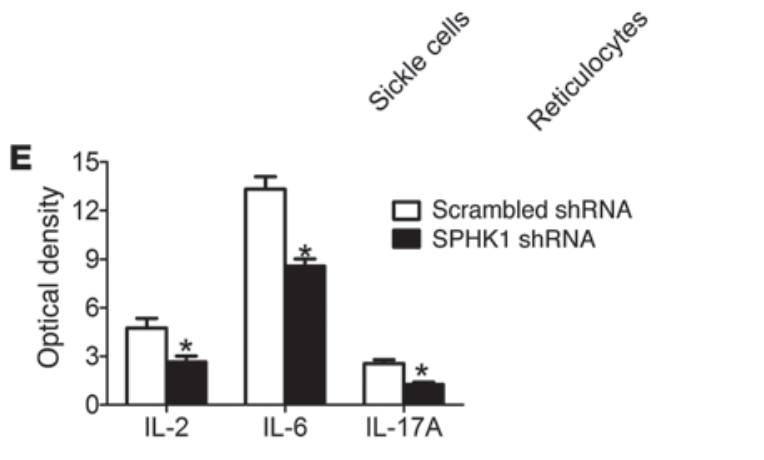

C

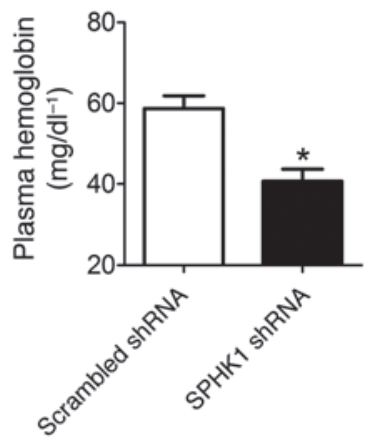

Figure 4

Specific knockdown of SPHK1 in HSCs in BMT SCD chimeras reduced sickling, hemolysis, inflammation, and prolonged life span of erythrocytes. (A) Blood smears of SCD chimeras with or without SPHK1 knockdown (original magnification, $\times 100$ ). (B) Percentages of sickle cells and reticulocytes were significantly reduced in the SCD chimeras with HSC-specific SPHK1 knockdown. (C-E) SPHK1 knockdown in HSCs (C) decreased plasma $\mathrm{Hb}$ levels, (D) prolonged life span of erythrocytes, and (E) reduced circulating cytokines in SCD chimeras. Values shown represent the mean \pm SEM $(n=6-11) .{ }^{*} P<0.05$ versus SCD chimeras with BMCs infected with recombinant lentivirus encoding scrambled shrna.

ure $2 \mathrm{~B}$ ). Moreover, the congestion, vascular damage, and necrosis in other tissues, including lungs and livers, were remarkably reduced in SCD mice with PF-543 treatment (Supplemental Figure 2A). Next, we found that vascular leakage in the lungs of PF-543-treated SCD mice was significantly decreased, as judged by reduced Evans blue staining (Supplemental Figure 2, C and D) and albumin concentrations in bronchial alveolar lavage fluid (Supplemental Figure 2E). Finally, we demonstrated that PF-543 treatment significantly increased survival rates under prolonged hypoxic conditions (Supplemental Figure 2F). Because acute lung inflammation and pulmonary congestion are well-accepted measures of vaso-occlusion, they are considered as major causative factors for hypoxia-induced death in SCD. Supporting this notion, we found that prolonged hypoxia induced substantial pulmonary congestion and elevation of multiple cytokines in the lung tissues compared with the SCD mice under normoxia (Supplemental Figure 2, G-I). Intriguingly, PF-543 treatment resulted in a significant decrease in hypoxia-induced pulmonary congestion and multiple inflammatory cytokines (Supplemental Figure 2, G-I), suggesting that the PF-543-mediated increase in survival rate under hypoxic conditions is likely due to reduced lung inflammatory storm and congestion. Altogether, our studies demonstrate that inhibition of elevated SPHK1 activity reduced tissue injury, disease progression, and hypoxia-induced early death.

Knockdown of SPHK1 in HSCs decreases sickling and reticulocytes in SCD chimeras by reducing S1P levels in the erythrocytes and circulation. To confirm our pharmacologic studies, we took a genetic approach to breed $S p h k^{-/-}$mice with SCD mice. Unfortunately, complete deletion of SPHK1 on the SCD background resulted in very few SCD/Sphk $k^{-/-}$pups, since S1P is essential for implantation for normal pregnancy (27). Thus, to overcome the difficulty of generating adult $\mathrm{SCD} / S p h k^{-/-}$mice, we took an alternative approach, using lentiviral vectors encoding specific shRNA to knockdown SPHK1 in bone marrow cells (BMCs) of SCD mice (Supplemental Figure 3). SPHK1 shRNA lentiviral vectors are a pool of concentrated, transduction-ready viral particles, containing 3 target-specific constructs that encode 19-25 nt (short hairpin) shRNA designed to specifically knockdown Sphk1 mRNA levels (28). This approach allowed us to genetically, specifically, and efficiently knockdown SPHK1 in the HSCs of SCD mice. For this purpose, we transfected BMCs isolated from SCD mice with recombinant lentivirus encoding shRNAs specific for SPHK1 or scrambled shRNA sequences (Santa Cruz Biotechnology Inc.). Initially, using flow cytometry, we found that $60 \%$ to $75 \%$ of BMCs expressed shRNA viral vector encoding EGFP. Furthermore, at this transduction efficiency, using quantitative PCR, we determined that the genetically modified cells contained approximately $3 \pm 0.5$ vector copies per cell. Following successful viral transduction, we transplanted the genetically modified BMCs from SCD mice to lethally irradiated WT recipients to generate SCD chimeras (Supplemental Figure 3). The life span of normal mouse erythrocytes is approximately 55 days. Thus, after 12 to 16 weeks after bone marrow transplantation (BMT), we used HPLC to determine the extent of erythrocyte chimerism by measuring the percentage of human sickle $\mathrm{Hb}$ (HbS) and normal mouse $\mathrm{Hb}$ in circulating erythrocytes of the transplant recipients. We found that nearly all of the circulating WT erythrocytes were replaced by SCD erythrocytes (characterized by the presence of $95 \%$ to $99 \%$ $\mathrm{HbS}$ ) derived from the SCD HSCs (Figure 3A). Similarly, using gel electrophoresis, we confirmed our HPLC results, showing that nearly all $\mathrm{Hb}$ in erythrocytes in SCD chimeras was human $\mathrm{HbS}$ derived from the Berkeley SCD mice (Figure 3A). Thus, these 
A Weight (g) $\quad 0.64 \pm 0.14$

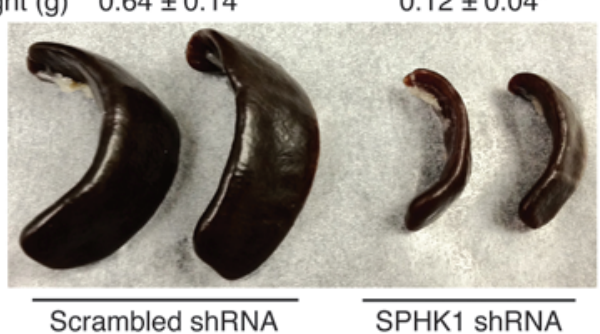

B
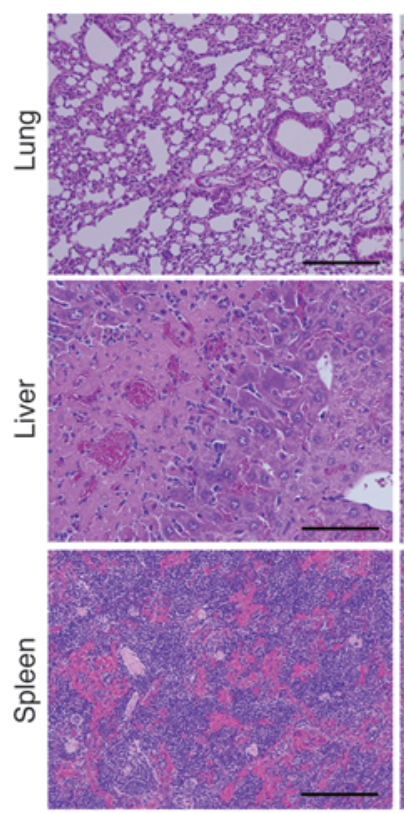

Scrambled shRNA

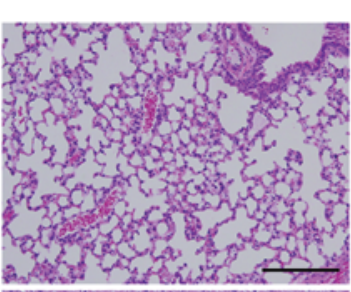

C Scrambled shRNA
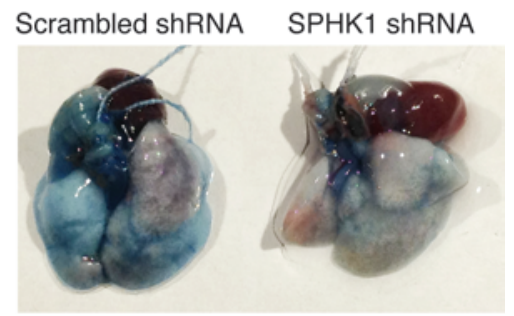

D

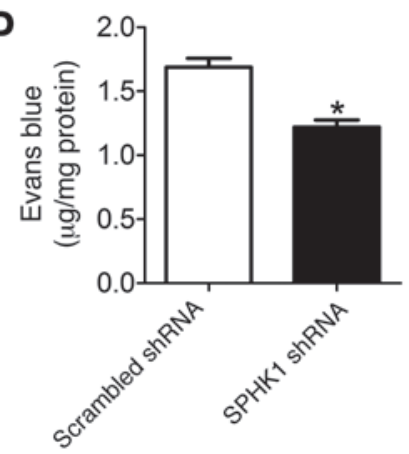

E

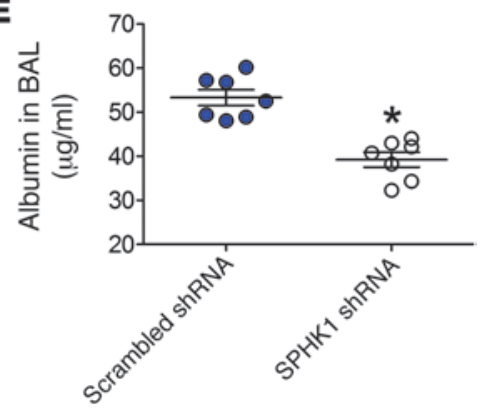

Figure 5

Specific knockdown of SPHK1 in HSCs in BMT SCD chimeras reduced splenomegaly and tissue damage. (A) Spleen size and (B) H\&E staining of spleens, livers, and lungs of SCD chimeras with HSC-specific SPHK1 knockdown and controls. (C) Representative Evans blue staining and (D) quantification of its concentration in the lungs of the control SCD chimeras and SCD chimeras with specific SPHK1 knockdown. (E) Albumin concentrations in bronchial alveolar lavage (BAL) fluid collected from the control SCD chimeras and SCD chimeras with specific SPHK1 knockdown. Values shown represent the mean \pm SEM $(n=6-11)$. ${ }^{*} P<0.05$ versus SCD chimeras with BMCs infected with recombinant lentivirus encoding scrambled shrna. Scale bar: $200 \mu \mathrm{M}$.

results indicate that we successfully generated chimeric mice in which the hematopoietic system was almost entirely derived from SCD BMCs. Next, at 12 to 16 weeks after transplantation, we confirmed that SPHK1 protein levels (Figure 3B) and enzyme activity (Figure 3C) in erythrocytes of WT mice that received SCD BMCs transduced with lentivirus encoding shRNA for SPHK1 were significantly reduced compared with the erythrocytes of WT mice transplanted with SCD BMCs transduced with lentivirus encoding scrambled shRNA. Additionally, we found that knockdown of SPHK1 in SCD HSCs resulted in reduction of erythrocyte and plasma S1P levels of the SCD chimeras (Figure 3, D and E). Consistent with our pharmacologic studies, we found that the size and shape of the erythrocytes were much more uniform and organized (Figure 4A) and that the percentages of sickled cells and reticulocytes in the SCD chimeras with specific knockdown of SPHK1 in hematopoietic cells were significantly reduced compared with those of the SCD chimera controls (Figure 4B). Thus, these quasi-genetic studies using SPHK1 knockdown in
BMT SCD chimeras allowed us to study erythrocyte function in the adult life and provide strong evidence supporting pharmacologic studies that increased SPHK1 activity is a key causative factor underlying increased S1P in erythrocytes and plasma of SCD Tg mice that thereby contributes to sickling.

Knockdown of SPHK1 in HSCs decreases intravascular hemolysis, prolongs erythrocyte life span, and reduces inflammation in SCD chimeras. Similar to PF-543 treatment, we found that lentiviral knockdown of SPHK1 in HSCs of SCD chimeras led to significantly decreased hemolysis, as demonstrated by decreased plasma $\mathrm{Hb}$ (Figure 4C). In addition to decreased hemolysis, decreased percentages of sickled cells, and decreased reticulocyte count, we also found that the half-life of erythrocytes in SCD chimeras increased from 2 days to 5 days following knockdown of SPHK1 (Figure 4D). Because of the antisickling and antihemolytic effects, CBC analysis showed that knockdown of SPHK1 in SCD chimeras significantly increased the total number of erythrocytes, $\mathrm{Hb}$ concentration, and hematocrit (Supplemental Table 2). Additionally, 

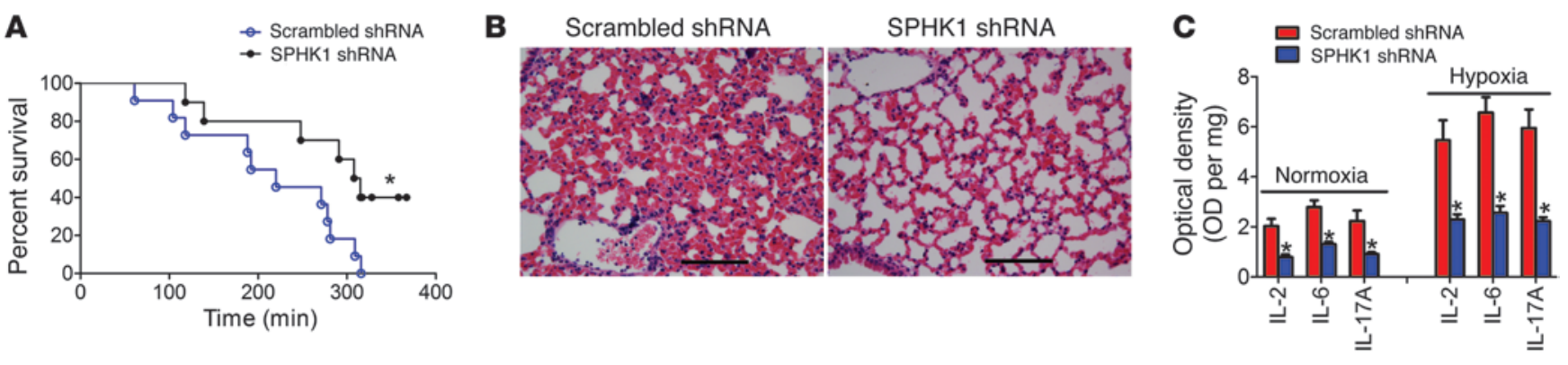

\section{Figure 6}

Specific knockdown of SPHK1 in HSCs in BMT SCD chimeras increased survival rates and reduced pulmonary congestion and inflammation under hypoxic conditions. (A) Knockdown of SPHK1 in HSCs prolonged survival rates of BMT SCD chimeras under hypoxic conditions with $8 \% \mathrm{O}_{2}$ concentration. (B and $\mathbf{C}$ ) Knockdown of SPHK1 in HSCs in SCD chimeras (B) reduced hypoxia-induced pulmonary congestion and (C) decreased elevation of multiple cytokines in the lung tissue. Values shown represent the mean \pm SEM $(n=6-9)$. ${ }^{*} P<0.05$ versus SCD chimeras with BMCs infected with recombinant lentivirus encoding scrambled shRNA. Scale bar: $200 \mu \mathrm{M}$.

erythrocyte distribution width was significantly reduced, reflecting more uniformly shaped cells, as seen in blood smear in SCD chimeras with specific SPHK1 knockdown (Supplemental Table 2). Consistently, mean cellular $\mathrm{Hb}$ was also improved in SCD Tg mice by knockdown of SPHK1 (Supplemental Table 2). Moreover, knockdown of SPHK1 reduced the total number of white blood cells to the normal range (Supplemental Table 2). Of note, both circulating lymphocytes and neutrophils were significantly reduced in SCD chimeras with SPHK1 knockdown (Supplemental Table 2). Consistently, circulating cytokines, including IL-2, IL-6, and IL-17, were also significantly decreased in these mice (Figure 4E). Altogether, these studies demonstrate that knockdown of SPHK1 in HSCs in SCD chimeras not only decreased sickling and reticulocytes, but also attenuated hemolysis, inflammation, and prolonged the life span of erythrocytes. As such, reduction of S1P by knockdown of SPHK1 specifically in SCD HSCs effectively increased erythrocyte number and total Hb content and decreased inflammation in SCD chimeras.

Knockdown of SPHK1 in HSCs attenuates splenomegaly and tissue injury at multiple sites and increases survival rates of SCD chimeras. Similar to our pharmacologic studies, we found that splenomegaly was substantially reduced in SCD chimeras with the specific knockdown of SPHK1 in HSCs compared with that in controls (from $0.64 \pm 0.14 \mathrm{~g}$ to $0.12 \pm 0.04 \mathrm{~g}$ ) (Figure 5A). Histological studies revealed that the specific knockdown of SPHK1 in SCD chimeras by shRNA prevented splenic damage, congestion, and necrosis (Figure 5B). Moreover, the congestion, vascular damage, and necrosis in other tissues, including lungs, livers, and spleens, were remarkably reduced in SCD chimeras with specific knockdown of SPHK1 in HSCs (Figure 5B). Semiquantitative analysis of histological changes demonstrated that knockdown of SPHK1 in HSCs in SCD chimeras resulted in significant improvement in comparison with that in controls (Supplemental Figure 4). More importantly, we found that the vascular leakage in the lungs of SPHK1 knockdown SCD chimeras was significantly decreased, as judged by reduced Evans blue staining (Figure 5, C and D) and albumin concentrations in bronchial alveolar lavage fluid (Figure 5E). Additionally, we further demonstrated that knockdown of SPHK1 SCD chimeras significantly increased survival rates under prolonged hypoxic conditions (Figure 6A) by attenuating hypoxia-induced pulmonary congestion and multiple inflammatory cytokines in the lungs (Figure 6, B and C). Altogether, our studies provide strong evidence for the detrimental effects of elevated SPHK1-mediated induction of S1P in tissue injury, disease progression, and early death.

Erythrocyte SPHK1 activity and S1P levels are elevated in patients with SCD and directly contribute to sickling in cultured primary buman sickle erythrocytes. To determine whether SPHK1 activity and S1P levels are also elevated in patients with SCD, we measured erythrocyte SPHK1 activity and determined S1P levels in both plasma and erythrocytes of control individuals and patients with SCD (for patient information see Supplemental Table 3). Similar to that in SCD mice, we found that erythrocyte SPHK1 activity and S1P levels in erythrocytes and plasma were significantly elevated in humans with SCD, suggesting that elevated SPHK1-mediated induction of S1P has a pathological role in patients with SCD (Figure 7).

Next, to determine the pathophysiological significance of elevated erythrocyte SPHK1 activity and increased erythrocyte S1P levels in sickling, we chose to expose cultured erythrocytes from humans with SCD to hypoxia to induce sickling in the presence or absence of PF-543, a potent specific SPHK1 inhibitor (24). We found that hypoxia significantly induced erythrocyte SPHK1 activity (Figure 8A) and resulted in the elevation of S1P (Figure $8 B$ ) in erythrocytes from patients with SCD. Pretreatment with PF-543 significantly inhibited hypoxia-induced SPHK1 activity (Figure 8A) and prevented the elevation of erythrocyte S1P in a dosage-dependent manner (Figure 8B). As such, pretreatment with PF-543 significantly reduced the percentage of sickled cells under hypoxic conditions in a dosage-dependent manner (Figure 8C). Moreover, to precisely determine the functional relationship of increased erythrocyte SPHK1 to sickling, we treated erythrocytes from patients with SCD with different oxygen pressures in the presence or absence of PF-543. We found that the percentage of sickle cells was inversely dependent on oxygen concentration (Figure 8D). Pretreatment with PF-543 significantly reduced the percentage of sickled cells under hypoxic conditions (Figure 8D). Thus, this study provides pharmacologic evidence that elevated erythrocyte SPHK1 underlies elevation of S1P and directly contributes to sickling in cultured human sickle erythrocytes.

$S 1 P$-induced sickling is independent of $S 1 P$ receptor activation. $\mathrm{S} 1 \mathrm{P}$ is a well-known signaling molecule, regulating diverse biological processes via activation of cell surface S1P receptors in an autocrine or paracrine manner and/or by interaction with key regulatory proteins 
A

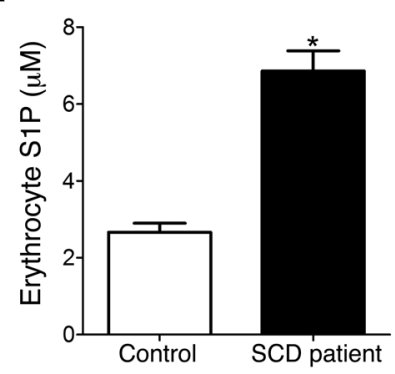

B

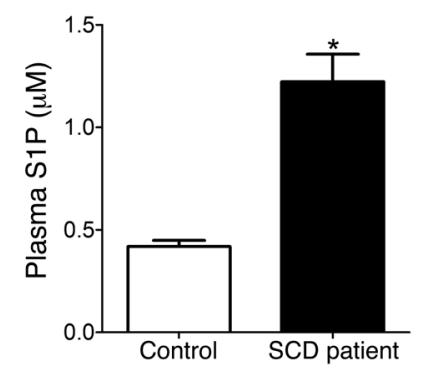

C

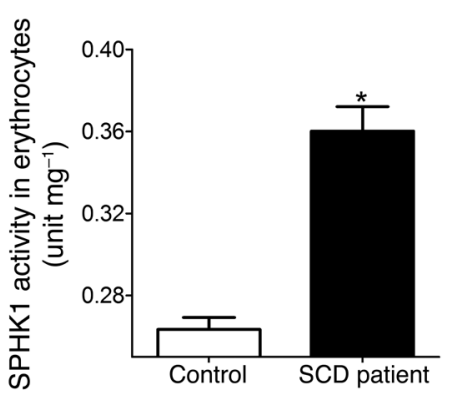

Figure 7

Erythrocyte SPHK1 activity and S1P levels in both erythrocytes and plasma are elevated in individuals with SCD. (A and B) Average S1P levels in (A) erythrocytes and (B) plasma from healthy volunteers (control, $n=14$ ) and patients with $\operatorname{SCD}(n=30)$. (C) Erythrocyte SPHK1 activity for healthy volunteers (control, $n=14$ ) and patients with $\operatorname{SCD}(n=30)$. Data are presented as the mean \pm SEM. ${ }^{*} P<0.05$ relative to the control individuals.

within cells $(16,29,30)$. In view of our finding that lowering erythrocyte S1P by inhibiting SPHK1 activity directly attenuated hypoxia-induced sickling in cultured human SCD erythrocytes (Figure 8, $\mathrm{C}$ and $\mathrm{D})$, we first tested the possibility that S1P-induced sickling is via activation of its surface receptors in an autocrine manner. To test this hypothesis, we treated human cultured sickle erythrocytes under hypoxic conditions with S1P receptor antagonists targeting S1PR1, S1PR2, and S1PR3. Unlike the SPHK1 inhibitor, none of the tested $\mathrm{S} 1 \mathrm{P}$ receptor antagonists inhibited hypoxia-induced sickling (Figure $8 \mathrm{E}$ ). Next, we treated primary cultured human sickle erythrocytes with 100 to $500 \mathrm{nM}$ exogenous S1P under different oxygen pressures to accurately assess the functional relationship of S1P to hypoxia-induced sickling. We found that S1P treatment failed to enhance sickling under hypoxic conditions, even at $500 \mathrm{nM}$, a concentration well beyond that required for $\mathrm{S} 1 \mathrm{P}$ receptor activation (Figure 8F). Thus, our results indicate that increased S1P generated by the activation of SPHK1 contributes to sickling by a mechanism that is independent of $\mathrm{S} 1 \mathrm{P}$ receptor activation.

\section{Discussion}

In this study, a nonbiased high-throughput metabolomic screen led us to find a previously unrecognized pathological role of elevated S1P in SCD. Using both pharmacologic approaches and quasi-genetic studies with SPHK1 knockdown in SCD BMT chimeras, we demonstrated that increased SPHK1 activity in SCD erythrocytes underlies elevated S1P within SCD erythrocytes and in plasma and contributes to sickling, hemolysis, inflammation, and multiple complications in SCD Tg mice. Human studies demonstrated that both SPHK1 and S1P are elevated in the erythrocytes of patients with SCD and treatment with SPHK1 inhibitors reduces hypoxia-mediated elevation of S1P and directly blocked hypoxia-induced sickling in cultured human SCD erythrocytes. Finally, we discovered that elevated S1P contributes to sickling independent of S1P receptor activation. Our discoveries provide evidence for both humans and mice that increased erythrocyte SPHK1 activity leads to S1P elevation and that excessive intracellular S1P is a previously unrecognized metabolite that contributes to sickling, the central pathogenesis of SCD, implicating novel therapeutic opportunities for the disease (Figure 8G).

$\mathrm{S} 1 \mathrm{P}$ is an important signaling molecule, which regulates diverse biological processes via activation of cell surface S1P receptors and/or by interaction with key regulatory proteins within cells
(16). For example, S1P is known to regulate egress of lymphocytes from thymus, spleen, and lymph nodes via $\mathrm{S} 1 \mathrm{P}$ receptor activation (10). More recent studies demonstrate that S1P is a key chemoattractant that induces myeloid progenitor cells to egress from bone marrow to peripheral blood (31). Additionally, $\mathrm{S} 1 \mathrm{P}$ functions as an intracellular signaling molecule to regulate activation of NF- $\mathrm{KB}$, a transcription factor well known to control inflammation (30). Besides the proinflammatory function, $\mathrm{S} 1 \mathrm{P}$ is also involved in tumor growth by promoting cell proliferation and angiogenesis (14). Moreover, S1P is reported to prevent endothelial leakage (13), acute kidney ischemic injury (32), and progression of multiple sclerosis (33) in mice. Although S1P is enriched in erythrocytes $(8,9)$, prior to our metabolomic screening, there was no evidence that S1P is elevated in the blood of individuals with SCD and no consideration given to the possibility that elevated S1P contributes to the pathogenesis of SCD.

Here, we have provided multiple lines of in vivo animal and in vitro human evidence supporting the role of S1P in promoting sickling. First, we provide in vivo pharmacologic evidence that inhibiting SPHK1 activity leads to a reduction of sickling by lowering erythrocyte S1P levels in SCD mice. We also provide quasi-genetic studies using SPHK1 knockdown in SCD BMT chimeras, showing that reducing erythrocyte SPHK1 protein levels results in decreased erythrocyte SPHK1 activity, S1P levels, and sickling. Translating our animal studies to humans, we showed that both SPHK1 and S1P are significantly elevated in patients with SCD. Moreover, we demonstrated that inhibition of SPHK1 activity by two structurally different inhibitors significantly reduced hypoxiainduced sickling in cultured human sickle cells. These studies provide strong cellular evidence that hypoxia-mediated elevated SPHK1 activity results in increased S1P production and directly contributes to sickling. Taken together, our results provide novel and compelling evidence in both humans and mice that increased erythrocyte SPHK1 activity results in elevated erythrocyte S1P and that elevated erythrocyte S1P contributes to sickling.

$\mathrm{S} 1 \mathrm{P}$ is a signaling molecule that functions extracellularly via its 5 surface receptors or intracellularly by interacting with other molecules (16). We have provided pharmacologic evidence that S1P receptor antagonists have no effects on hypoxia-induced sickling (Figure 8E). S1P is known to activate all of its receptors at low nM concentration (16). However, exogenous S1P treatment even at 500 nM did not enhance sickling under hypoxia conditions (Figure 8F). Thus, neither S1P receptor antagonism nor S1P treatment had any 


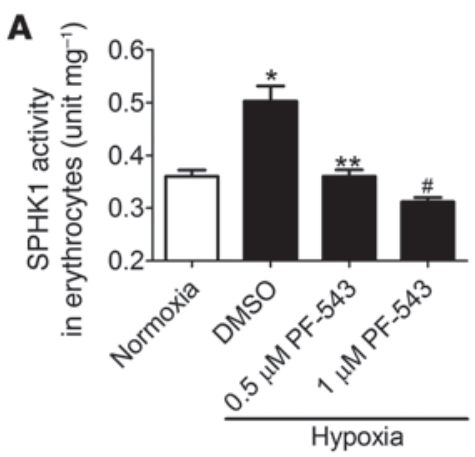

D

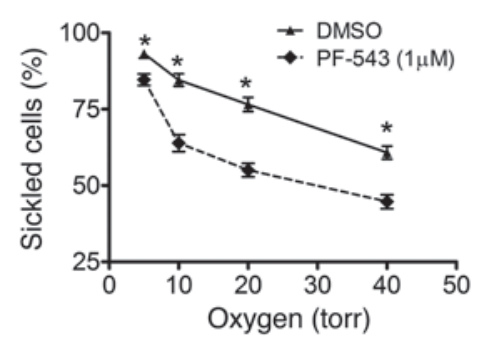

$\mathbf{F}$

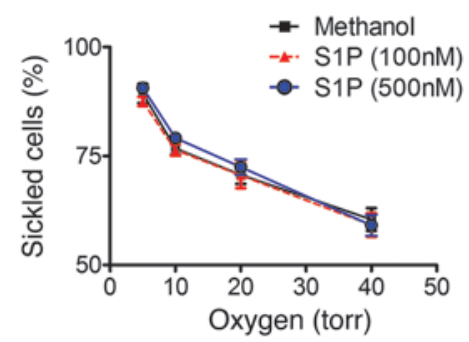

B

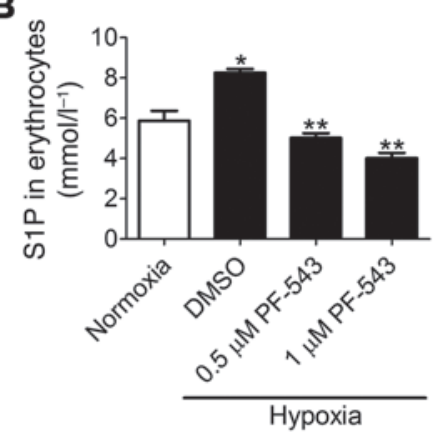

E

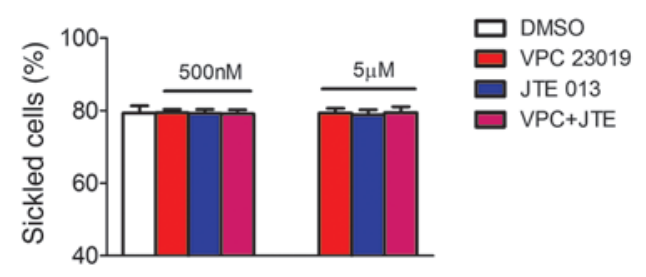

G

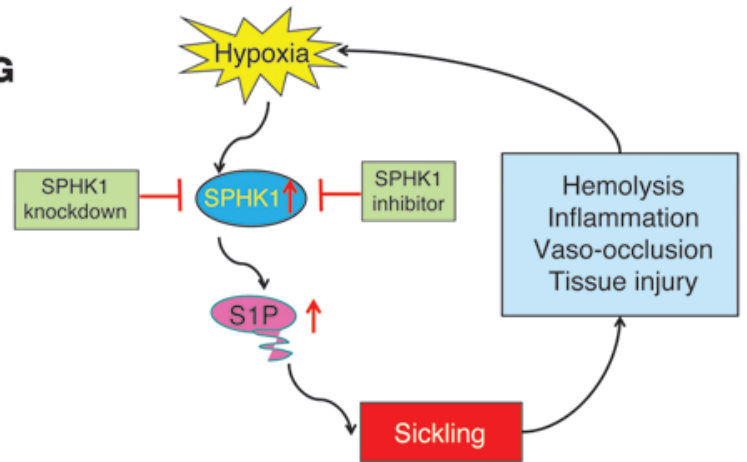

Figure 8

SPHK1-mediated elevation of S1P contributes directly to hypoxia-induced sickling in cultured human sickle erythrocytes independent of S1P receptor activation. (A-C) Pretreatment of cultured primary erythrocytes isolated from patients with SCD with PF-543 inhibited hypoxia-mediated induction of (A) SPHK1 activity, (B) S1P production, and (C) sickling in a dosage-dependent manner. (D) Changes in the percentage of sickled cells in erythrocytes isolated from patients with SCD, following exposure to different hypoxic conditions in the absence or presence of PF-543 treatment. (E) S1P contributes to sickling independent of S1P receptor activation. S1P receptor antagonists (VPC, S1P 1 and $\mathrm{S}_{1} \mathrm{P}_{3}$ receptor antagonist; JTE, S1P 2 receptor antagonist) did not reduce hypoxia-induced sickling. (F) Exogenous S1P (100 to $500 \mathrm{nM}$ ) did not enhance hypoxia-induced sickling in cultured human sickle erythrocytes under different hypoxia conditions. Data are presented as the mean \pm SEM. ${ }^{*} P<0.05$ relative to the samples under normoxic conditions, ${ }^{* *} P<0.05$ relative to untreated hypoxia samples, ${ }^{P} P<0.05$ relative to PF-543-treated samples at lower concentration $(n=5-6)$. (G) Under hypoxic conditions, increased S1P, due to elevated erythrocyte SPHK1 activity, contributes to sickling independent of its receptor activation. Because erythrocytes store the highest amount of S1P $(8,9)$, the hemolysis associated with sickling unleashes massive amounts of this pleiotropic signaling molecule with widespread detrimental effects, including inflammation and tissue injury at multiple sites. Without interference, increased sickling, hemolysis, inflammation, and tissue injury function as a malicious cycle, leading to more severe hypoxia and further elevation of erythrocyte SPHK1 activity and S1P levels. The use of SPHK1 inhibitors to lower S1P levels reduces sickling and represents a potentially important novel mechanism-based therapy for SCD.

effect on hypoxia-induced sickling in human cultured erythrocytes. In contrast, we found that inhibition of SPHK1 by its potent inhibitors directly reduced erythrocyte S1P levels and subsequent sickling under different hypoxia conditions (Figure 8D), indicating the SPHK1-induced elevation of S1P-mediated sickling is independent of cell surface receptor activation. Thus, these studies imply that S1P functions intracellularly to induce sickling and motivate future functional and structural studies to determine the molecular basis underlying S1P inducted sickling.
Our unbiased metabolic screen and follow-up studies revealed a detrimental role of elevated S1P in the setting of SCD. The debilitating consequences are explained by our unexpected finding that elevated S1P promotes sickling, hemolysis, tissue damage at multiple sites, and shortened life span. Taken together, our discovery adds a significant new chapter to erythrocyte pathology by revealing S1P as a bioactive lipid contributing to sickling independent of S1P receptors. We believe hypoxia is an initial trigger to induce erythrocyte SPHK1 activity, resulting in subsequent elevation of 
erythrocyte S1P. Because erythrocytes are the largest reservoir of $\operatorname{S1P}(8,9)$ (in part due to the absence of S1P-degrading enzymes), the hemolysis associated with SCD unleashes massive amounts of this pleiotropic signaling molecule into plasma, with potentially widespread detrimental effects, including excessive inflammation and tissue injury at multiple sites (Figure 8G). Thus, without interference, SPHK1 mediated increased erythrocyte S1P-induced sickling and hemolysis function in a malicious cycle, facilitating further elevation of S1P, sickling, hemolysis, inflammatory cascade, and exacerbation of tissue injury at multiple sites. However, in the presence of a SPHK1 inhibitor or genetic knockdown of SPHK1 in HSCs, which lowers S1P levels, the positive feedback loop is interrupted, thereby resulting in remarkable antisickling, antihemolytic, and antiinflammatory effects that subsequently attenuate tissue damage and disease progression. Thus, we have revealed that SPHK1-mediated S1P elevation in SCD erythrocytes is a key contributor to sickling in SCD and that SPHK1 inhibition can attenuate both acute and chronic sickling events and disease progression (Figure 8G). Thus, the work reported here could be the foundation leading to future human trials and a possible therapy for SCD.

\section{Methods}

\section{Metabolomic profiling}

Nonbiased metabolomic screening of whole blood of control and SCD mice ( $n=6$ for each) was performed using LC/GC-MS, as described previously (6). Specifically, a Thermo Fisher linear ion-trap mass spectrometer with Fourier transform and a Mat-95 XP mass spectrometer were used to analyze 7,000 named metabolites. The LC/MS POS platform was optimized for compounds that show positive ionization as described previously (6). There were 251 small metabolites detected in the circulation of both the controls and SCD mice. The combinations of groups were analyzed using Welch's 2 -sample $t$ test, following log transformation and imputation with minimum observed values for each compound. $P<0.05$ was considered significant.

\section{Blood collection and preparation from bumans and mice}

Approximately $7 \mathrm{ml}$ blood was withdrawn from forearm veins of normal individuals and patients with SCD. One $\mathrm{ml}$ of the blood was collected in a $1.5-\mathrm{ml}$ tube containing 17 USP units of sodium heparin and mixed well. The plasma and packed erythrocytes were stored at $-80^{\circ} \mathrm{C}$ for $\mathrm{S} 1 \mathrm{P}$ and sphingosine kinase activity measurement (see below). Four ml blood was collected with EDTA as an anticoagulant and used for morphological study, oxygen equilibrium curve measurement, and CBC. Two $\mathrm{ml}$ blood with no anticoagulant was used for electrolyte and chemical panel assay. Mouse blood was collected in a similar way, except for smaller volumes.

\section{Sample preparation and S1P measurement by LC/MS analysis}

Sample preparation. The sample preparation was carried out in a 96-well plate on ice. Plasma or erythrocyte samples $(50 \mu \mathrm{l})$ were mixed with $20 \mu \mathrm{l}$ of an internal standard solution $(1,000 \mathrm{ng} / \mathrm{ml} \mathrm{S1P-d}$ ) (Avanti Polar Lipids) and $500 \mu \mathrm{l}$ of methanol. The plate was capped, vortexed for 1 minute at room temperature, and centrifuged for 10 minutes at $3,000 \mathrm{~g}$ at room temperature. $300 \mu \mathrm{l}$ aliquot of supernatant was transferred to a new plate for LC-MS/MS analysis. Both S1P and the internal standard (S1P-d7) were purchased from Avanti Polar Lipids, with purity over $99 \%$. Stock solution $(1.25 \mathrm{mg} / \mathrm{ml})$ of S1P was prepared by dissolving $2.53 \mathrm{mg} \mathrm{S} 1 \mathrm{P}$ in $2.00 \mathrm{ml}$ of a mixture of water/methanol/n-butanol $/ 6$ $\mathrm{N} \mathrm{HCl} \mathrm{(50:25:25:1).} \mathrm{The} \mathrm{same} \mathrm{solvent} \mathrm{mixture} \mathrm{was} \mathrm{also} \mathrm{used} \mathrm{to} \mathrm{dissolve}$ the internal standard. The calibration standards were prepared at con- centrations of 2,000, 1,600, 500, 250, 100, 25, 10, and $5 \mathrm{ng} / \mathrm{ml}$. Quality control samples were prepared from pooled plasma, whole blood, or erythrocytes. The S1P levels in the prepared samples were accurately analyzed by the means detailed below.

Chromatography. An Agilent 1290 Infinity UPLC system equipped with binary solvent pumps, a refrigerated autosampler, and a column heater (set at $40^{\circ} \mathrm{C}$ ) was used for liquid chromatography using a reverse-phase column (Waters ACQUITY UPLC BEH C18, $1.7 \mu \mathrm{m}, 2.1 \times 100 \mathrm{~mm}$ ), with mobile phase A consisting of $5 \mathrm{mM}$ ammonium formate in water containing $1 \%$ formic acid and mobile phase B consisting of $5 \mathrm{mM}$ ammonium formate in methanol containing $1 \%$ formic acid. An isocratic condition of $90 \%$ of mobile phase B was used for elution. The total run time was 3 minutes, and the flow rate was $0.4 \mathrm{ml}$ per minute. An aliquot of $2 \mu \mathrm{l}$ of the final extraction solution was injected for each sample. The eluent was directly introduced into a mass spectrometer by electrospray.

Mass spectrometry. An ABSciex API 5500 QTrap mass spectrometer was used in this study. The instrument was operated in positive multiple reaction monitoring (MRM) mode controlled by Analyst 1.5.1 software. Ion pairs 380.2/264.3 and 387.2/271.3 were selected for MRM of the analyte and internal standard, respectively. Ion spray voltage was $5 \mathrm{kV}$; source temperature was $500^{\circ} \mathrm{C}$; curtain gas was set at $40 \mathrm{l} / \mathrm{min}$; and the nebulizer and desolvation gas flow rates were set at $60 \mathrm{l} / \mathrm{min}$. Declustering potential was set at $70 \mathrm{~V}$, and entrance potential was set at $6.0 \mathrm{~V}$. The collision energy was set at $24 \mathrm{eV}$, and collision cell exit potential was set at $14 \mathrm{~V}$. The resolutions of Q1 and Q3 were set at unity.

\section{In vivo treatment of SCD mice with PF-543 and DMSO}

Eight- to twelve-week-old SCD mice were anesthetized by intraperitoneal injection with $200 \mu \mathrm{l}$ of $2.5 \%$ avertin. Osmotic minipumps (Alza) were implanted subcutaneously in the nape of the neck. PF-543 (Millipore) was delivered at a rate of $0.93 \mathrm{mg} / \mathrm{kg}$ body weight per day into mice for 28 days. Control mice received DMSO.

\section{Lentivirus production and bone marrow transduction}

Recombinant lentiviruses were produced in $293 \mathrm{~T}$ cells by transient transfection of lentivirus packaging plasmids, including 4 plasmids expressing GagPol (pHDM-Hgpm2), Tat (pHDM-tat1b), Rev (pRC-CMV-rev1B), and VSV-G ( $\mathrm{pHDM}$ VSV-G), and a pool of 3 recombinant lentiviral vectors, each encoding shRNA specific for SPHK1 (Santa Cruz Biotechnology Inc., sc-45446) or scrambled shRNA (Santa Cruz Biotechnology Inc., sc-108080). Cell culture media containing viruses was collected 3 times within 72 hours after transfection and concentrated by centrifugation (34). Concentrated virus stocks had a titer greater than $2 \times 10^{8}$ per $\mathrm{ml}$ and were stored at $-80^{\circ} \mathrm{C}$ before $\mathrm{BMC}$ transduction.

BMCs were isolated from SCD Tg mice by flushing isolated femurs and tibias with DMEM (35). Single-cell suspension was prepared by passing the cells through a cell strainer and 25-gauge needle. BMCs were plated in a 12-well plate, and viral transductions were performed by incubating $8 \times 10^{6} \mathrm{BMCs}$ cells with $4 \times 10^{8}$ recombinant lentiviruses produced as described above in DMEM medium (Thermo Scientific) containing $8 \mu \mathrm{g} / \mathrm{ml}$ polybrene. The plate was sealed with paraffin film and centrifuged at $1,000 \mathrm{~g}$ for 2 hours at $30^{\circ} \mathrm{C}$. After centrifugation, the plate was incubated at $37^{\circ} \mathrm{C}$ for 2 hours. BMCs were collected in 1.5 -ml tubes and centrifuged at $1,000 \mathrm{~g}$ for 10 minutes and resuspended in PBS with 0.25 \% BSA. Cells were placed on ice for injection.

\section{Irradiation and BMT}

The day before irradiation, C57BL/6 mice (12 to 14 weeks of age) were treated with neomycin at $2 \mu \mathrm{g} / \mathrm{ml}$ in drinking water, as described previously (35). The next day, mice were exposed to $5 \mathrm{~Gy}$ body irradiation with a gamma irradiator (Gammacell, MDS Nordion). Four hours later, the mice were exposed to the same dose of irradiation. BMCs transduced by 
recombinant lentivirus, as described above, were injected into the tail veins of irradiated mice $\left(1 \times 10^{6} \mathrm{BMCs}\right.$ per mouse $)$. After BMT, the mice were injected intraperitoneally with $1 \mu \mathrm{g} / \mathrm{kg}$ body weight erythropoietin per day for 3 days and treated with $2 \mu \mathrm{g} / \mathrm{ml}$ neomycin in drinking water for 2 weeks. Mice were sacrificed 12-16 weeks later for experiments.

\section{Measurement of SPHK1 activity and protein level in the erythrocytes of mice}

Mouse blood was collected with EDTA. The blood was centrifuged at $240 \mathrm{~g}$ for 10 minutes at room temperature, followed by aspiration of plasma and white blood cell-containing interface. Erythrocyte pellets were lysed in a buffer containing $20 \mathrm{mM}$ PIPES, $150 \mathrm{mM} \mathrm{NaCl}, 1 \mathrm{mM}$ EGTA, 1\% v/v Triton $\mathrm{X}-100,1.5 \mathrm{mM} \mathrm{MgCl}_{2}$, and $1 \mathrm{mM}$ Na-orthovanadate ( $\left.\mathrm{pH} 7.4\right)$. The total protein concentration was measured with a Protein Assay Kit (Bio-Rad). One part of cell lysates was used for SPHK1 activity measurement by SPHK1 activity assay (Echelon Bioscience). The other portion of cell lysates was used for Western blot analysis to quantify SPHK1 protein levels. Approximately $100 \mu \mathrm{g}$ total protein was run on $12 \%$ SDS-PAGE gels and then transferred to nitrocellulose membrane. The membrane was blocked with 5\% nonfat milk (Bio-Rad) and incubated with anti-SPHK1 antibody (LifeSpan BioSciences) and secondary antibody (Santa Cruz Biotechnology Inc.), respectively. The protein band was visualized by being developed in ECL solution (Pierce) and exposed to $\mathrm{x}$-ray film.

\section{Statistics}

All data are expressed as the mean \pm SEM. Data were analyzed for statistical significance using GraphPad Prism 4 software (GraphPad Software). Two-tailed Student's $t$ tests (paired or unpaired as appropriate) were applied in 2-group analysis. Differences between the means of multiple groups were compared by 1-way analysis of variance, followed by a Turkey's multiple comparisons test. A $P$ value of less than 0.05 was considered significant.

\section{Study approval}

Human subjects. Patients with SCD in the steady state were identified by hematologists on the faculty of the University of Texas Medical School at Houston and Brody School of Medicine of East Carolina University. Patients participating in this study had no blood transfusion for at least 6 months before blood samples were collected. Control subjects were of African descent and were free of hematological disease. Signed informed consent was obtained from all subjects. The research protocols were approved by the University of Texas Health Science Center at Houston and Brody School of Medicine of East Carolina University Committees for the Protection of Human Subjects. Detailed information regarding patients and controls can be found in Supplemental Table 3 and the Supplemental Methods.

Mice. SCD Berkeley Tg mice, exclusively expressing HbS, were purchased from The Jackson Laboratory. The SCD Tg mice were produced by breeding homozygous males with heterozygous female mice. Sphk $k^{-1-}$ mice were generated by Richard L. Proia (NIH) and provided by his group (27). Four-week-old mice were genotyped by PCR. C57BL/6 mice used as controls were purchased from Harlan. Animal care was in accordance with University of Texas Health Science Center at Houston and NIH guidelines. Research protocols were reviewed and approved by the University of Texas Health Science Center at Houston Animal Welfare Committee.

\section{Acknowledgments}

This work was supported by NIH grants HL119549 (to Y. Xia), DK077748 (to Y. Xia), DK083559 (to Y. Xia), P01HL114457 (Project 3 to Y. Xia), and HL095820 (to A.-L. Tsai) as well as American Heart Association grants 10GRNT3760081 and 12IRG9150001 (to Y. Xia), China Scholarship Council 2009637520 (to Weiru Zhang), and NIH/NHLBI grant HL103186 (to O.Y. Abdulmalik).

Received for publication December 5, 2013, and accepted in revised form March 27, 2014.

Address correspondence to: Yang Xia, Department of Biochemistry and Molecular Biology, University of Texas Medical School at Houston, 6431 Fannin St., Houston, Texas 77030, USA. Phone: 713.500.5039; Fax: 713.500.0652; E-mail: yang.xia@uth.tmc.edu.
1. Madigan C, Malik P. Pathophysiology and therapy for haemoglobinopathies. Part I: sickle cell disease. Expert Rev Mol Med. 2006;8(9):1-23.

2. Urbinati F, Madigan C, Malik P. Pathophysiology and therapy for haemoglobinopathies. Part II: thalassaemias. Expert Rev Mol Med. 2006;8(10):1-26.

3. Ingram VM. Sickle-cell anemia hemoglobin: the molecular biology of the first "molecular disease" - the crucial importance of serendipity. Genetics. 2004;167(1):1-7.

4. Strasser BJ. Perspectives: molecular medicine. "Sickle cell anemia, a molecular disease". Science. 1999;286(5444):1488-1490.

5. Steinberg MH. Sickle cell anemia, the first molecular disease: overview of molecular etiology, pathophysiology, and therapeutic approaches. ScientificWorldJournal. 2008;8:1295-1324.

6. Evans AM, DeHaven CD, Barrett T, Mitchell M, Milgram E. Integrated, nontargeted ultrahigh performance liquid chromatography/electrospray ionization tandem mass spectrometry platform for the identification and relative quantification of the small-molecule complement of biological systems. Anal Chem. 2009;81(16):6656-6667.

7. Weckwerth W. Metabolomics: an integral technique in systems biology. Bioanalysis. 2011;2(4):829-836.

8. Ito $\mathrm{K}$, et al. Lack of sphingosine 1-phosphatedegrading enzymes in erythrocytes. Biochem Biophys Res Commun. 2007;357(1):212-217.

9. Hanel P, Andreani P, Graler MH. Erythrocytes store and release sphingosine 1-phosphate in blood. Faseb J. 2007;21(4):1202-1209.

10. Pappu R, et al. Promotion of lymphocyte egress into blood and lymph by distinct sources of sphingosine-1phosphate. Science. 2007;316(5822):295-298.

11. English D, et al. Sphingosine 1-phosphate released from platelets during clotting accounts for the potent endothelial cell chemotactic activity of blood serum and provides a novel link between hemostasis and angiogenesis. FASEB J. 2000;14(14):2255-2265.

12. Nagahashi M, et al. Sphingosine-1-phosphate produced by sphingosine kinase 1 promotes breast cancer progression by stimulating angiogenesis and lymphangiogenesis. Cancer Res. 2012;72(3):726-735.

13. Camerer E, et al. Sphingosine-1-phosphate in the plasma compartment regulates basal and inflammation-induced vascular leak in mice. J Clin Invest. 2009;119(7):1871-1879.

14. English D, Brindley DN, Spiegel S, Garcia JG. Lipid mediators of angiogenesis and the signalling pathways they initiate. Biochim Biophys Acta. 2002;1582(1-3):228-239.

15. English D, Garcia JG, Brindley DN. Plateletreleased phospholipids link haemostasis and angiogenesis. Cardiovasc Res. 2001;49(3):588-599.

16. Spiegel S, Milstien S. Sphingosine-1-phosphate: an enigmatic signalling lipid. Nat Rev Mol Cell Biol. 2003;4(5):397-407.

17. Sankala HM, et al. Involvement of sphingosine kinase 2 in $\mathrm{p}^{53}$-independent induction of 21 by the chemotherapeutic drug doxorubicin. Cancer Res. 2007;67(21):10466-10474.

18. Kobayashi N, Kobayashi N, Yamaguchi A, Nishi T. Characterization of the ATP-dependent sphingosine 1-phosphate transporter in rat erythrocytes. J Biol Chem. 2009;284(32):21192-21200.

19. Kihara A, Igarashi Y. Production and release of sphingosine 1-phosphate and the phosphorylated form of the immunomodulator FTY720. Biochim Biophys Acta. 2008;1781(9):496-502.

20. Paszty C. Transgenic and gene knock-out mouse models of sickle cell anemia and the thalassemias. Curr Opin Hematol. 1997;4(2):88-93.

21. Paszty C, et al. Transgenic knockout mice with exclusively human sickle hemoglobin and sickle cell disease. Science. 1997;278(5339):876-878.

22. Maceyka M, Harikumar KB, Milstien S, Spiegel S. Sphingosine-1-phosphate signaling and its role in disease. Trends Cell Biol. 2012;22(1):50-60.

23. Paugh SW, et al. A selective sphingosine kinase 1 inhibitor integrates multiple molecular therapeutic targets in human leukemia. Blood. 2008; 112(4):1382-1391.

24. Ackers GK, Benesch RE, Edalji R. Effects of inositol hexasulfate on the oxygen affinity of hemoglobin: verification of the integral function theory of thermodynamic linkage. Biochemistry. 1982;21(5):875-879.

25. Kato GJ, Gladwin MT, Steinberg MH. Deconstructing sickle cell disease: reappraisal of the role of hemolysis in the development of clinical subphe- 
notypes. Blood Rev. 2007;21(1):37-47.

26. Puneet $P$, et al. SphK1 regulates proinflammatory responses associated with endotoxin and polymicrobial sepsis. Science. 2010;328(5983):1290-1294.

27. Mizugishi K, et al. Maternal disturbance in activated sphingolipid metabolism causes pregnancy loss in mice. J Clin Invest. 2007;117(10):2993-3006.

28. Yao C, et al. Co-administration phenoxodiol with doxorubicin synergistically inhibit the activity of sphingosine kinase-1 (SphK1), a potential oncogene of osteosarcoma, to suppress osteosarcoma cell growth both in vivo and in vitro. Mol Oncol. 2012;6(4):392-404

29. Hait NC, et al. Regulation of histone acetylation in the nucleus by sphingosine-1-phosphate. Science.
2009;325(5945):1254-1257.

30. Alvarez SE, et al. Sphingosine-1-phosphate is a missing cofactor for the E3 ubiquitin ligase TRAF2. Nature. 2010;465(7301):1084-1088.

31. Ratajczak MZ, et al. Novel insight into stem cell mobilization-plasma sphingosine-1-phosphate is a major chemoattractant that directs the egress of hematopoietic stem progenitor cells from the bone marrow and its level in peripheral blood increases during mobilization due to activation of complement cascade/membrane attack complex. Leukemia. 2011;24(5):976-985.

32. Awad AS, et al. Chronic sphingosine 1-phosphate 1 receptor activation attenuates early-stage diabetic nephropathy independent of lymphocytes. Kidney
Int. 2011;79(10):1090-1098

33. Webb M, et al. Sphingosine 1-phosphate receptor agonists attenuate relapsing-remitting experimental autoimmune encephalitis in SJL mice. J Neuroimmunol. 2004;153(1-2):108-121.

34. Mostoslavsky G, Kotton DN, Fabian AJ, Gray JT, Lee JS, Mulligan RC. Efficiency of transduction of highly purified murine hematopoietic stem cells by lentiviral and oncoretroviral vectors under conditions of minimal in vitro manipulation. Mol Ther. 2005;11(6):932-940.

35. Hsu LL, et al. Hemolysis in sickle cell mice causes pulmonary hypertension due to global impairment in nitric oxide bioavailability. Blood. 2007; 109(7):3088-3098. 